\title{
Antimicrobial agents and gonorrhoea: therapeutic choice, resistance and susceptibility testing
}

\author{
Catherine A Ison
}

Introduction: Neisseria gonorrhoeae, the causative agent of gonorrhoea is a particularly well adapted pathogen that has continued to evolve mechanisms to evade treatment with antimicrobial agents.

Therapeutic choice: The choice of antibiotic for use in the first-line treatment of gonorrhoea should be made with knowledge of the susceptibility of the isolates of $N$ gonorrhoeae to be encountered.

Resistance: High-level resistance to penicillin and tetracycline in $N$ gonorrhoeae is plasmidmediated and a major therapeutic problem. Penicillinase-producing $N$ gonorrhoeae, first described in 1976, have now spread worldwide and tetracycline-resistant $N$ gonorrhoeae, described in 1985, are becoming increasingly prevalent. Chromosomal resistance to penicillin is low-level and affects a range of antibiotics. High-level resistance to spectinomycin has been sporadic and has not limited its use whereas the emergence of resistance to ciprofloxacin will have a significant impact on its use for gonorrhoea.

Susceptibility testing: A variety of methods are available including disc diffusion, breakpoint agar dilution technique, E-test and determination of the minimum inhibitory concentration (MIC). The choice of methodology will depend on the number and type of isolates and the facilities available for testing.

Discussion: Surveillance programmes to monitor levels of antibiotic resistant isolates are essential to ensure therapeutic success.

(Genitourin Med 1996;72:253-257)

Keywords: gonorrhoea; antimicrobial agents; resistance

\section{Introduction}

Neisseria gonorrhoeae are inherently susceptible to antibiotics but due to continual use, particularly of penicillin, both chromosomally and plasmid-mediated resistance has developed. Treatment for gonorrhoea is often given after a presumptive diagnosis of intracellular Gram negative cocci detected on a Gram smear has been made and before the organism is isolated and results of susceptibility testing obtained. To delay therapy would allow further transmission of the organisms. In order that the therapy given is effective the choice of antibiotic should be made with knowledge of the susceptibility patterns of the gonococcal population in that geographical location. Surveillance of susceptibility can predict the outcome of therapy, detect the emergence of resistant strains and monitor drifts in susceptibility to the treatment of choice. A variety of methods are available including disc diffusion, breakpoint agar dilution technique, E-test and determination of the minimum inhibitory concentration (MIC).

This paper attempts to review microbiological factors which will influence the choice of antibiotic for therapy; types of resistance encountered, prevalence of resistant isolates, methodology for determining susceptibility and approaches to testing of gonococcal isolates.

\section{Antimicrobial resistance}

Chromosomal resistance Chromosomal resistance to penicillin is low-level and the result of the additive effect of mutations at multiple loci, including pen $A, m$ tr and pen $B .{ }^{1}$ pen $A$ encodes for penicillin-binding protein 2 (PBP-2), and a mutation which results in a single amino-acid change confers decreased affinity for penicillin. ${ }^{2}$ The $m t r$ locus consists of an operon, $m t r C D E$, which controls susceptibility to a range of hydrophobic antibiotics and detergents by an efflux system which actively removes the antibiotic from the cell. ${ }^{34}$ The function of the locus, pen $B$, is unknown but it is either very closely linked or part of the por gene that encodes for the major porin of $N$ gonorrhoeae. It is likely that mutations occur within this gene that alter cell wall permeability to penicillin. The additive effect of these mutations can increase the MIC from $\leqslant 0.06 \mathrm{mg} / \mathrm{l}$ in a susceptible strain to $\geqslant 1$ $\mathrm{mg} / \mathrm{l}$ with an increased chance of therapeutic failure. Reduced susceptibility to the cephalosporins such as cefuroxime, cefotaxime and ceftriaxone appears to be conferred by mutations in same loci..$^{5}$ Low-level resistance to antibiotics such as tetracycline and erythromycin is also the result of chromosomal mutations in loci that are closely linked to penB. These isolates are termed chromosomallymediated resistant $N$ gonorrhoeae (CMRNG).

Resistance to spectinomycin in $N$ gonorrhoeae differs from penicillin in that it occurs in a single step. It is high-level and results in an increase in MIC from 8-16 mg/l for isolates prior to therapy to $\geqslant 256 \mathrm{mg} / \mathrm{l}$ for isolates post-treatment. Mutations on the chromosome affect binding of the antibiotic to the ribosome. ${ }^{6}$

Ciprofloxacin is a fluoroquinolone and is 
highly active against $N$ gonorrhoeae. This antibiotic has only been in routine use for the treatment of gonorrhoea for about five years. ${ }^{7}$ Sporadic reports of therapeutic failure emerged quite quickly in isolates with MICs of $\geqslant 0.12 \mathrm{mg} / 1^{8-11}$ but it is only in recent months that the emergence of high-level resistance in isolates with MICs of $16 \mathrm{mg} / 1$ has caused concern. ${ }^{12-15}$

The mechanism of resistance to ciprofloxacin in other bacteria results from mutations in the DNA gyrase genes or changes in cell wall permeability. Evidence from spontaneous mutants of $N$ gonorrhoeae with increasing MICs to ciprofloxacin indicated that moderate levels of resistance result from aminoacid changes in gyr $A$ whereas mutants with high levels of resistance had changes in both gyrA and the topoisomerase IV gene, parC. ${ }^{16}$ Alterations in these genes has now been associated with resistance in clinical isolates. ${ }^{1718}$ In vitro it is easy to obtain spontaneous mutants by selection on increasing concentrations of ciprofloxacin. These laboratory mutants with MICs of $16 \mathrm{mg} / 1$ were obtained before the isolation of similar strains from therapeutic failures. This confirms that use of low or inadequate dosages for treatment will ultimately select for resistant isolates.

Plasmid-mediated resistance Plasmid-mediated resistance to penicillin was first documented in 1976. Two reports occurred simultaneously, one of an isolate which originated from Africa and was isolated in Liverpool $^{19}$ and the other from the Far East which was isolated in the USA. ${ }^{20}$ The two strains carried plasmids of $3.2 \mathrm{MDa}$ (African) and $4.4 \mathrm{MDa}$ (Asian) respectively and both encoded for a TEM-1 type beta-lactamase, the smaller plasmid having a deletion in a nonfunctional region of the plasmid. ${ }^{21}$ Transfer of penicillinase plasmids can occur between strains of $N$ gonorrhoeae by conjugation but requires the presence of a plasmid of 24.5 $\mathrm{MDa}$ in the donor to mobilise the transfer. ${ }^{22}$ The $24.5 \mathrm{MDa}$ plasmid was found among isolates carrying the $4.4 \mathrm{MDa}$ penicillinase plasmid and these strains quickly spread to other parts of the world. However, the conjugative plasmid was not found in combination with isolates carrying the $3 \cdot 2 \mathrm{MDa}$ penicillinase plasmid until $1981^{23}$ and hence dissemination of these strains occurred more slowly. Penicillinase-producing $N$ gonorrhoeae (PPNG) are now disseminated worldwide. PPNG carrying plasmids of differing size have also been reported in recent years. ${ }^{24-27}$ They are all related to the original plasmids and encode for the TEM-1 beta-lactamase but, as yet, have not spread so widely. Penicillinase plasmids are thought to have originated from Haemophilus $s p$ but their spread is species restricted.

High-level plasmid-mediated resistance to tetracycline in $N$ gonorrhoeae was first reported in $1985 .{ }^{28}$ It is due to the acquisition of the tet $M$ determinant by the conjugative plasmid (24.5 MD) of $N$ gonorrhoeae resulting in a plasmid of $25 \cdot 2 \mathrm{MDa}$. This plasmid is selfmobilisable and can move between strains of
$N$ gonorrhoeae and other genera. The DNA sequence of the tet $M$ determinant from TRNG appears to vary and can be subdivided into two types ${ }^{29} 30$ which correlate with the location of the original isolates, USA and the Netherlands. ${ }^{29}$ Tetracycline-resistant $N$ gonorrhoeae (TRNG) are likely to spread more quickly than PPNG because of the presence of tet $M$ plasmids in other flora found in the genital tract may act as a reservoir, the ability for self-transfer between different genera and selection by use of tetracycline for chlamydial infection or for gonorrhoea in some parts of the world.

\section{Prevalence of antibiotic resistant Neisseria gonorrhoeae}

Penicillin resistant strains of $N$ gonorrhoeae are more prevalent in developing countries where effective antibiotics are unavailable or too expensive and contact tracing procedures are not developed. Most of the data have been collected from sentinel studies and the prevalence of chromosomal and plasmid-mediated resistance to penicillin can be as high as $80 \%$ of the isolates tested. In many other countries reporting systems are in place but still produce misleading results due to under reporting. For instance in England and Wales reporting of resistant isolates to the Communicable Disease Surveillance Centre, PHLS is voluntary and consequently the number of resistant strains is probably an underestimate. The most accurate data are available from the USA $^{31}$ and Australia ${ }^{32} 33$ where good surveillance programmes exist. The Gonococcal Isolate Surveillance Project (GISP) in the USA showed $15.0 \%$ of isolates tested were PPNG and $15.4 \%$ were CMRNG in 1993 (JS Knapp, personal communication). The Australian surveillance programme found in 1995 that $7.5 \%$ of isolates tested were PPNG and $8.3 \%$ CMRNG (J Tapsall, personal communication). Although the true prevalence of resistance to penicillin is unknown in most industrialised countries it is regarded as most prevalent among isolates from patients attending clinics in the major cities, much of which is from imported infections. ${ }^{34}$ Concerns over the increase in penicillin worldwide, both chromosomal and plasmid-mediated, led the World Health Organization to change the recommendations for first line therapy from penicillin to spectinomycin, ceftriaxone or ciprofloxacin unless the gonococcal population is known to be susceptible to penicillin.

Resistance to spectinomycin differs from that to penicillin. Although there have been several documented episodes of spectinomycin resistant $N$ gonorrhoea $e^{35}{ }^{36}$ it has not spread or limited the use of the antibiotic. Spectinomycin is not a widely used treatment for gonorrhoea but will be particularly useful if resistance develops to the newer alternatives such as ceftriaxone and ciprofloxacin. Therapeutic failure with ceftriaxone is undocumented whereas there is an increasing number of reports of ciprofloxacin resistant isolates. ${ }^{8-15}$ These quinolone-resistant $N$ gonorrhoeae 
Table 1 Recommended concentrations of antibiotics for susceptibility testing of $N$ gonorrhoeae ${ }^{*}=4$ units)

\begin{tabular}{lccl}
\hline \multicolumn{3}{c}{ Method } & \\
\cline { 2 - 4 } Antibiotic & $\begin{array}{l}\text { Disc diffusion } \\
(\mu g)\end{array}$ & $\begin{array}{l}\text { Breakpoint } \\
(m g / l)\end{array}$ & $\begin{array}{l}\text { MIC } \\
(\mathrm{mg} / \mathrm{l})\end{array}$ \\
\hline Penicillin & $2.4^{\star}$ & 0.5 & $0.015-8$ \\
Spectinomycin & 100.0 & 32.0 & $8-64$ \\
Ceftriaxone & 0.1 & 0.12 & $0.002-0.12$ \\
Ciprofloxacin & 0.1 & 0.12 & $0.002-0.5$ \\
\hline
\end{tabular}

(QRNG) have been isolated in a number of countries including the UK, USA and Australia but mostly originate from countries of the South-Eastern Pacific or China. The Australian surveillance programme identified high-level resistance (MIC, $\geqslant 1 \mathrm{mg} / \mathrm{l}$ ) in $1.9 \%$ of isolates in 1995 (J Tapsall, personal communication) reflecting importation from countries where use of quinolones has not been controlled.

\section{Susceptibility testing}

Chromosomal resistance Detection of chromosomal resistance can be achieved by using disc diffusion, determination of the minimum inhibitory concentration (MIC), breakpoint agar dilution technique or the E-test. There are no universally accepted guidelines for testing $N$ gonorrhoeae but different approaches predominate; in North America, susceptibility testing is performed to NCCLS guidelines ${ }^{37}$; in Australia a standardised method ${ }^{32} 33$ is used and there are also recommended methods from the World Health Organisation. ${ }^{38}$

A number of factors influence the results of all the methods used for in vitro susceptibility testing: medium used, inoculum and concentration of antibiotic tested. The medium used varies from enriched bases such as GC agar base or Columbia agar to sensitivity test agars such as Diagnostic Sensitivity Test (DST) agar and Isosensitest agar. A range of supplements has also been employed including IsoVitaleX, Kellogg's supplement, haemoglobin or lysed horse blood. The effect of the composition of the medium and supplement on the results obtained with antibiotics such as penicillin, spectinomycin and cephalosporins is not significant. However, MICs obtained for tetracycline and erythromycin differ with the choice of medium used. ${ }^{3940}$ The inoculum used has varied from $10^{3}-10^{5}$ colony forming units (cfu). When the higher inocula are used isolates appear more resistant. For standardisation between laboratories, an inoculum of $10^{4}$ cfu is generally used.

Of the methods available, disc diffusion is

Table 2 Pattern of susceptibility of WHO control strains for susceptibility testing of $N$ gonorrhoeae. WHO-E is a penicillinase-producing strain

\begin{tabular}{lllll}
\hline & \multicolumn{2}{l}{ Strain } & & \\
\cline { 2 - 5 } Antibiotic & WHO-A & WHO-B & WHO-C & WHO-D \\
\hline Penicillin & S & IR & IR & R \\
Spectinomycin & R & S & S & S \\
Ciprofloxacin & S & S & S & RS \\
Cetriaxone & S & S & S & S \\
\hline
\end{tabular}

$\mathrm{S}=$ Susceptible, $\mathrm{R}=$ Resistant $\mathrm{IR}=$ Intermediate resistance, $\mathrm{RS}=$ Reduced susceptibility. the most commonly used. In addition to the factors discussed above the choice of disc content will affect the results obtained. The NCCLS guidelines recommend the use of high content discs but in Europe and Australia lower content discs are usually used. Table 1 shows the antibiotic concentration of discs which are commercially available and have been recommended by the WHO Collaborating Centre in Copenhagen (Inga Lind, personal communication).

Agar incorporation techniques are used both to determine the full MIC or to categorise isolates into susceptible or resistant using the breakpoint technique where only one or two concentrations of antibiotic are used..$^{41}$ Three media have been widely used for these purposes; GC agar base supplemented with $1 \%$ IsoVitaleX (or Kellogg's supplements), DST agar or Isosensitest agar supplemented with $5 \%$ lysed horse blood in addition to $1 \%$ IsoVitaleX. The concentration suitable for breakpoints and the range for MIC determination of antibiotics commonly used for the treatment of gonorrhoea when DST agar is used is shown in table 1 . The concentrations of antibiotic used for breakpoints should be chosen in combination with the medium because GC agar base generally gives an endpoint one dilution higher than sensitivity test agar. For example, penicillin concentration should be changed from $0.5 \mathrm{mg} / 1$ (MIC $\geqslant 1.0$ $\mathrm{mg} / \mathrm{l})$ to $1.0 \mathrm{mg} / 1$ (MIC $\geqslant 2.0 \mathrm{mg} / \mathrm{l}$.

The recently developed E-test (AB Biodisk, Solna, Sweden) may be a simpler alternative to the conventional method for MIC determination. This method uses a plastic carrier strip with a predefined continuous exponential antibiotic gradient on one side which is applied to the surface of a previously seeded agar plate. The MIC is recorded at the point when the zone interacts with the strip. Recent evaluations of the E-test have shown the test to compare well with conventional MICs. ${ }^{42} 43$

The results obtained by any of the above methods will vary between and within laboratories because of the factors already discussed. The use of a panel of strains of known susceptibility is probably the most efficient means of controlling the variation. Such a panel is available from the WHO Collaborating centre and their susceptibility patterns are shown in table 2 . This panel, together with an isolate showing reduced susceptibility to ciprofloxacin, is a good basis for a control panel.

Plasmid-mediated resistance Plasmid-mediated resistance is simpler to detect. Penicillinase production in $N$ gonorrhoeae is most commonly detected using the chromogenic cephalosporin (Nitrocefin, Unipath Laboratories) test. ${ }^{44}$ The action of the penicillinase changes the colour of the reagent from yellow to a pink/red. Colonies from a primary isolation plate are transferred to filter paper, a drop of the reagent added and a result obtained in a few seconds. The starch-iodine method for detection of penicillinase is more time consuming to perform but is efficient and less expensive than the Nitrocefin reagent.

Plasmid-mediated resistance to tetracycline 
can be detected using screening methods of either no zone of inhibition around a $10 \mathrm{ug}$ disc or growth on GC agar containing $10 \mathrm{mg} / 1$ tetracycline. Confirmation of the presence of the tet $M$ determinant is achieved using hybridisation with a suitable probe. However, this is not possible in most laboratories and the screening tests have been shown to be good predictors of the presence of the tet $M$ determinant. ${ }^{45}$ For laboratories with appropriate facilities, TRNG can be detected using the polymerase chain reaction..$^{30}$

\section{Discussion}

The conventional approach to susceptibility testing is to test every strain on the day of isolation. However, this may be two or more days after the patient has been treated and if the first-line treatment in use gives very low therapeutic failure it will give very little information to the clinician. For instance, in the United Kingdom spectinomycin, ciprofloxacin and ceftriaxone are known to give therapeutic success approaching $100 \%$. A large number of consecutive isolates will be tested to detect the few potentially resistant isolates. It may be more informative to determine the patterns of susceptibility over time so that the likelihood of cure can be predicted. Surveillance programmes to achieve this test consecutive isolates either from every patient or from patients over a selected time period. The possible disadvantage of such an approach is that detection of the emergence of a highly resistant strain may be delayed or missed. This is a particular problem for clinics whose patients acquire their infections from geographically diverse areas. As an adjunct to surveillance, screening for high-level resistance is advisable. This can be simple to perform and inexpensive. PPNG can be detected with Nitrocefin reagent, TRNG and QRNG by subculture onto agar containing $10 \mathrm{mg} / \mathrm{l}$ tetracycline and $1 \mathrm{mg} / 1$ ciprofloxacin respectively. All results can be obtained within 24 hours of isolation of the organism.

Many laboratories prefer to perform full susceptibility testing on a daily basis. However, the choice of methodology for testing $N$ gonorrhoeae is difficult. Disc diffusion has been widely used and is a successful method if the isolate is fully susceptible or resistant but, because the technique is dependent on the many variables discussed above, the definition of a zone size that denotes reduced susceptibility can be difficult. The breakpoint technique is an attractive alternative to disc diffusion. It has the advantage of giving a predicted range for the MIC and can be used with a multipoint inoculator so that up to 20 strains can be tested at one time. For laboratories that only encounter isolates of $N$ gonorrhoeae intermittently it may be more appropriate to use the E-test. Determination of the full MIC should not be attempted by routine laboratories and should be reserved for reference centres or for use in surveillance programmes.

$N$ gonorrhoeae is a versatile and successful pathogen of humans and continues to evolve mechanisms to evade our attempts at eradication with antimicrobial agents. In order to maintain therapeutic success it is essential that trends in susceptibility are monitored through surveillance programmes.

I thank Dr Joan Knapp, Atlanta, USA and Professor John Tapsall, Sydney, Australia for providing recent data for this review.

1 Sparling PF, Sarubbi FA, Blackman E. Inheritance of lowlevel resistance to penicillin, tetracycline and chloramphenicol in Neisseria gonorrhoeae. $\mathcal{f}$ Bacteriol 1975;124: $740-9$.

2 Spratt BG. Hybrid penicillin-binding proteins in penicillinresistant strains of Neisseria gonorrhoeae. Nature 1988;332:173-6.

3 Pan W, Spratt BG. Regulation of the permeability of the gonococcal cell envelope by the mtr system. Mol Microbiol 1994;11:769-75.

4 Hagman KE, Pan W, Spratt BG, Balthazar JT, Judd RC, Shafer WS. Resistance of Neisseria gonorrhoeae to antimicrobial hydrophobic agents is modulated by the mtrCDE crobial hydrophobic agents is modulated by the

5 Ison CA, Bindayna KM, Woodford N, Gill MJ, Easmon CSF. Penicillin and cephalosporin resistance in gonococci. Genitourin Med 1990;66:351-6.

6 Maness MJ, Foster GC, Sparling PF. Ribosomal resistance to streptomycin and spectinomycin in Neisseria gonorrhoeae. ₹ Bacteriol 1974;120:1293-9.

7 Echols RM, Heyd A, O'Keefe BJ, Schacht P. Single-dose ciprofloxacin for the treatment of uncomplicated gonorrhoea: a worldwide summary. Sex Transm Dis 1994;21: 345-52.

8 Gransden WR, Warren C, Phillips I. 4-quinolone-resistant Neisseria gonorrhoeae in the United Kingdom. $f$ Med Microbiol 1991;34:23-7.

9 Knapp JS, Ohye R, Neal SW, Parekh MC, Higa H, Rice $R J$. Emerging in vitro resistance to quinolones in penicillinase-producing Neisseria gonorrhoeae strains in Hawaii. nase-producing Neisseria gonorrhoeae strains

10 Tapsall JW, Shultz TR, Lovett R, Munro R. Failure of 500 mg ciprofloxacin therapy in male urethral gonorrhoea. Med f Aust 1992;156:143.

$11 \mathrm{Kam} \mathrm{KM}, \mathrm{Lo} \mathrm{KK}, \mathrm{Ng} \mathrm{KYH}$, Cheung MM. Rapid decline in penicillinase-producing Neisseria gonorrhoeae in Hong Kong associated with emerging 4-fluoroquinolone resistance. Genitourin Med 1995;71:141-4.

12 Turner A, Gough KR, Jephcott AE, McClean AN. Importation into the United Kingdom of a strain of Neisseria gonorrhoeae resistant to penicillin, ciprofloxacin and tetracycline. Genitourin Med 1995;71:265-6.

13 Birley H, MacDonald P, Carey P, Fletcher J. High-level ciprofloxacin resistance in Neisseria gonorrhoeae. Genitourin Med 1994;70:292-3.

14 Tapsall JW, Limnios EA. Thacker C, Donovan B, Lynch SD, Kirby LJ, et al. High-level quinolone resistance in Neisseria gonorrhoeae: a report of two cases. Sex Transm Neisseria gonorrhoea

15 CDC, Fluoroquinolone resistance in Neisseria gonorrhoeaeColorado and Washington, 1995. MMWR 1995;44: $761-5$

16 Belland RJ, Morrison SG, Ison C, Huang WM. Neisseria gonorrhoeae acquires mutations in analogous regions of gyr $A$ and $p a r C$ in fluoroquinolone-resistant isolates. Mol Microbiol 1994;14:371-80.

17 Deguchi $T$, Yasuda $M$, Asama $M$, Tada $K$, Iwata $H$, Komeda $\mathrm{H}$, et al. DNA gyrase mutations in quinoloneresistant clinical isolates of Neisseria gonorrhoeae. Antimicrob Agents Chemother 1995;39:561-3.

18 Deguchi T, Yasuda M, Nakano M, Ozeki S, Ezaki T, Saito I, et al. Quinolone-resistant Neisseria gonorrhoeae: Correlations of the gyrA subunit of DNA gyrase and the Correlations of the gyrA subunit of DNA gyrase and the
ParC subunit of topoisomerase IV with antimicrobial susParC subunit of topoisomerase IV with antimicrobial sus-
ceptibility profiles. Antimicrob Agents Chemother 1996;40: ceptibility

19 Phillips I. Beta-lactamase-producing, penicillin-resistant gonococcus. Lancet 1976;ii: 656-7.

20 Ashford WA, Golash RG, Hemming VG. Penicillinaseproducing Neisseria gonorrhoeae. Lancet 1976;ii:657-8.

21 Elwell LP, Roberts M, Mayer LW, Falkow S. Plasmidmediated beta-lactamase production in Neisseria gonorrhoeae. Antimicrob Agents Chemother 1977;11:528-33.

22 Roberts MC, Falkow S. Conjugal transfer of R plasmids in Neisseria gonorrhoeae. Nature 1977;266:630-1.

23 Embden JDA van, Klingeren B van, Dessens-Kroon $\mathbf{M}$, Wijngaarden LJ van. Emergence in The Netherlands of penicillinase-producing gonococci carrying "Africa" plaspenicilinase-producing gonococci carrying "Africa" plasmid in co $1981 ; \mathrm{i}: 938$.

24 Embden JDA van, Dessens-Kroons $M$, Klingeren B van. A new beta-lactamase plasmid in Neisseria gonorrhoeae. $\mathcal{f}$ Antimicrob Chemother 1985;15:247-50.

25 Gouby A, Bourg G, Raamuz M. Previously undescribed 6.6 kilobase $R$ plasmid in penicillinase-producing Neisseria gonorrhoeae. Antimicrob Agents Chemother 1986; 29:1095-7. 
26 Yeung K-H, Dillon JR, Pauze M, Wallace E. A novel 4.9 kilobase plasmid associated with an outbreak of penicill nase-producing Neisseria gonorrhoeae. F Infect Dis 1986, 153:1162-5.

27 Brett $M$. A novel gonococcal beta-lactamase plasmid. $f$ Antimicrob Chemother 1989;23:653-4.

28 Morse SA, Johnson SR, Biddle JW, Roberts MC. Highlevel tetracycline resistance in Neisseria gonorrhoeae is
result of acquisition of streptococcal tetM determinant. result of acquisition of streptococcal tetM dete

29 Gascoyne-Binzi DM, Heritage J, Hawkey PM. Nucleotide sequences of the tet $(M)$ genes from the American and Dutch type tetracycline resistance plasmid of Neisseria gonorrhoeae. F Antimicrob Chemother 1993;32:667-76.

30 Xia M, Pang Y, Roberts MC. Detection of two groups of $25 \cdot 2 \mathrm{MDa}$ TetM plasmids by polymerase chain reaction of the downstream region. Mol Cell Probes 1995;9: 327-32.

31 Schwarcz SK, Zenilman JM, Schnell D, Knapp JS, Hook III EW, Thompson $S$, et al. National surveillance of antimicrobial resistance in Neisseria gonorrhoeae. $\mathcal{J} A M A$ 1990;264:1413-7.

32 Australian Gonococcal Surveillance Programme. Penicillin sensitivity of gonococci isolated in Australia, 1981-6.

33 Members of the Australian Gonococcal Surveillance Programme. Penicillin sensitivity of gonococci in Australia: Development of Australian gonococcal surveillance programme. Br f Venereal Dis 1984;60:226-30

34 Ison CA, Woodford PJ, Roope NS, Easmon CSF. Surveillance of antibiotic resistance in Neisseria gonorrhoeae. In: Conde-Glez CJ, Morse S, Rice P, Sparling F, Calderon E (eds). Pathobiology and Immunobiology of Neisseriaceae. Pub Instituto Nacional De Salud Publica. p $71-6$

35 Ashford WA, Potts DW, Adams HHJU, English JC Johnson SR, Biddle JW, Thornsberry C, Jaffe HW. gonorrhoeae. Lancet 1981;ii:1035-7.
36 Easmon CSF, Forster GE, Walker GD, Ison CA, Harris JRW, Munday PE. Spectinomycin as initial treatment for gonorrhoea. BMF 1984;289:1032-4.

37 National Committee for Clinical I aboratory Standards. Methods for dilution antimicrobial susceptibility tests for bacteria that grow aerobically. NCCLS Document M7A2, Vol 10, No 8. Villanova, Pa USA:NCCLS 1990.

38 World Health Organization. Bench-level manual for sexually transmitted diseases. WHO/VDT/89.443.

39 Woodford N, Ison CA. The effect of media on antimicrobial susceptibility testing of Neisseria gonorrhoeae. $\mathcal{f}$ Antimicrob Chemother 1988;22:463-71.

40 Dillon JR, Tostowaryk W, Pauze M. Effects of different media and methods of inoculum preparation on results of antimicrobial susceptibility testing of Neisseria gonorrhoeae by agar dilution. Antimicrob Agents Chemother 1987;31:1744-9.

41 Ison CA, Branley NS, Kirtland K, Easmon CSF Surveillance of antibiotic resistance in clinical isolates of Neisseria gonorrhoeae. BMF 1991;303:1307.

42 van Dyck E, Smet $H$, Piot $P$. Comparison of E-test with agar dilution for antimicrobial susceptibility testing of Neisseria gonorrhoeae. $f$ Clin Microbiol 1994;32: of Neisseria $1586-8$.

43 Yeung K-H, Ng L-K, Dillon JR. Evaluation of E test for testing antimicrobial susceptibilities of Neisseria gonorrhoeae isolates with different growth media. F Clin Microbiol 1993:3053-5.

44 O'Callaghan CH, Morris A, Kirby SM, Shingler AH. Novel method for the detection of beta-lactamases by using a chromogenic cephalosporin substrate. Antimicrob Agents Chemother 1972;1:283-8.

45 Ison CA, Tekki N, Gill MJ. Detection of the tetM determinant in Neisseria gonorrhoeae. Sex Transm Dis 1993;20: 329-33.

46 Members of the Australian gonococcal surveillance programme. Penicillin sensitivity of gonococci in Australia: Development of Australian gonococcal surveillance programme. $\mathrm{Br} \mathcal{F}$ Venereal Dis 1984;60:226-30. 